\title{
Mediastinal Non-Hodgkin Lymphoma with Bilateral Pleural Involvement - Role of Sonography
}

\author{
Rishabh Surinder Gupta ${ }^{1}$, Suresh Vasant Phatak ${ }^{2}$, Amruta Dinesh Varma ${ }^{3}$, Sharvari Shashikant Gulve ${ }^{4}$ \\ 1,2,3,4 Department of Radio-Diagnosis, Jawaharlal Nehru Medical College, \\ DMIMS-DU, Sawangi, Wardha, Maharashtra, India.
}

\section{INTRODUCTION}

Mediastinal form of lymphoma can present in two ways, one as disseminated disease leading to one component being present in mediastinum and second as a primary disease. Lymphomas constitute about $15 \%$ of all primary masses in mediastinum, and about $45 \%$ of primary masses in anterior mediastinum of children. ${ }^{1} 10 \%$ of mediastinal lymphomas are primary (i.e. isolated mediastinal involvement not related to any systemic disease) and the major part is Hodgkin lymphomas ( $\sim 60$ \%). ${ }^{2}$ Primary mediastinal lymphoma has histology of the diffuse type. The most common type of pathology is PDL-D (Poorly Differentiated Lymphocytic, Diffuse) with convoluted morphology. The diagnosis of lymphoma is confirmed on pathological histology.

Morphology is used to distinguish between Hodgkin Lymphoma (HL), characterised by the Reed-Sternberg cells (40\%), and Non-Hodgkin Lymphomas (NHL) (60\%). These are classified according to architectural criteria (follicular or diffuse) and morphological criteria (small or large cells). ${ }^{3}$

Mediastinal lymphadenopathy is most common in Hodgkin lymphoma and most frequently involves anterior and paratracheal lymph nodes in a contiguous pattern. It also involves in decreasing order of frequency the hilar, subcarinal, peri diaphragmatic, paraesophageal, and internal mammary nodes. ${ }^{4}$ Vital intra-thoracic structures can be commonly compressed. At presentation this entity usually is seemingly localized, but often it implies a presence of disseminated disease. ${ }^{5}$ Patients of mediastinal lymphoma are asymptomatic most of the times. Constitutional symptoms is most common systemic manifestations of lymphoma. Large mediastinal masses produce symptoms like dyspnoea, cough and retrosternal chest pain. Mediastinal lymphomas originate from the thymus or lymph nodes; thus the anterior and middle mediastinum are most common locations. ${ }^{6}$ The anterior mediastinal and para-tracheal nodes are the regions that are most frequently involved. Hilar node enlargement is often accompanied by mediastinal node enlargement. Posterior mediastinal involvement is uncommon. Small, perivascular lymphomas of the supraaortic branches are particularly diagnosed with suprasternal sonography. Suprasternal sonography can provide important additional information in patients with problematic CT (Computed Tomography) findings, particularly children and patients with allergies to contrast media. Consistency and monitoring the treatment of mediastinal tumours is also aided by suprasternal sonography. Finally, the suprasternal approach is useful for USG (Ultra-Sound Guided) biopsies of mediastinal tumours. ${ }^{7}$
Corresponding Author: Dr. Suresh Vasant Phatak. Department of Radio-Diagnosis, Jawahralal Nehru Medical College, DMIMS- DU, Sawangi,

Wardha, Maharashtra, India.

E-mail: suresh_phatak@yahoo.com

DOI: $10.14260 /$ jemds/2020/769

How to Cite This Article:

Gupta RS, Phatak SV, Varma AD, et al. Mediastinal non-Hodgkin lymphoma with bilateral pleural involvement - role of sonography. J Evolution Med Dent Sci 2020;9(46):3509-3511, DOI: $10.14260 / \mathrm{jemds} / 2020 / 769$

Submission 17-07-2020,

Peer Review 02-10-2020,

Acceptance 08-10-2020,

Published 16-11-2020.

Copyright (c) 2020 Rishabh Surinder Gupta et al. This is an open access article distributed under Creative Commons Attribution License [Attribution 4.0 International (CC BY 4.0)] 


\section{PRESENTATION OF CASE}

An 18-year-old male presented with complaints of retrosternal chest pain, cough, dyspnoea in Medicine OPD (Out-Patient Department). The patient was advised radiograph of chest, which revealed mediastinal mass with widening and was referred to radio diagnosis department for ultrasound examination of thorax.

It revealed massive bilateral pleural effusion [Figure 1]. Suprasternal USG with high frequency probe revealed multiple centimetric hypoechoic lymph nodes around the major vessels of mediastinum [Figure 2]. Suprasternal and parasternal approaches were used to accurately visualize the location, number, echogenicity and extent of mediastinal lymph nodes. Pleural fluid cytology confirmed our findings of Non-Hodgkin lymphoma (acute lymphoblastic leukaemia).

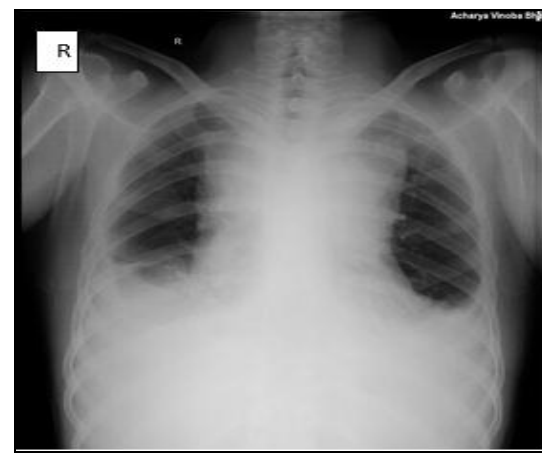

Figure 1. Frontal X-Ray of Chest Showing Mediastinal Widening and Bilateral Pleural Effusion

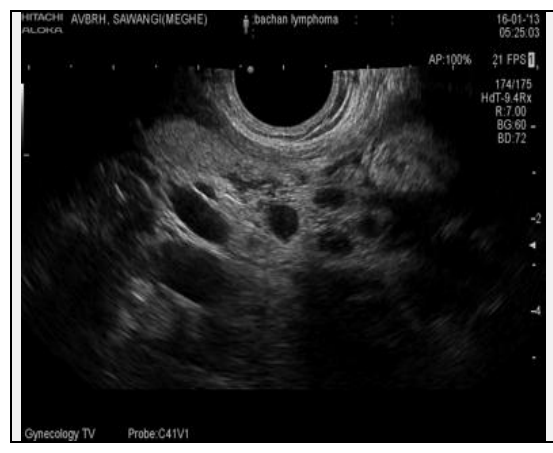

Figure 2. Suprasternal USG Showing Centrimetric Lymph nodes around the Great Vessels of Mediastinum.

\section{DISCUSSION}

Mediastinal form of lymphoma can present in two ways, one as disseminated disease leads to one component being present in mediastinum and second as a primary disease. Patients of mediastinal lymphoma are asymptomatic most of the times. Constitutional symptoms are most common systemic manifestations of lymphoma. Mediastinal lymphomas have a predilection for the anterior and middle mediastinum because they originate from the thymus or lymph nodes. Sonography is useful adjunct to other imaging modalities in mediastinal lymphomas.

$80 \%$ of HLs present as thoracic lymphomas in form of mediastinal lymph node involvement. Only $5 \%$ of NHL patients show pulmonary involvement. Adenomegalies are frequently seen in the initial stage of pulmonary HL. Isolated pulmonary lesion is seen in NHL. Pleural involvement is seen as thickening of pleura. Isolated or multiple pleural nodules occurs in $15-16 \%$ of NHLs, mostly as disseminated forms and sometimes as recurring forms. Direct lesions or lymphatic obstruction originating from lymph nodes can both lead to effusions. Lymph node form is the most well-known form of lymphoma and it is a classic form of Hodgkin lymphoma and low-grade NHLs. A pathological lymph node is the one that has a short axis of more than $1 \mathrm{~cm} .^{3}$ Many diagnostic procedures are available for use in the correct identification of the variety of neoplasms, cysts, inflammatory processes, and aneurysms that may present as a mass in mediastinum. Mediastinal mass evaluation follows a logical sequence starting from simpler and going towards more complicated techniques. ${ }^{8}$ Mediastinal sonography is an effective, precise and low-cost imaging method, with sensitivity higher than conventional radiographs, for the detection of mediastinal masses. ${ }^{9}$ The superiority of mediastinal sonography over chest radiography is due to its capability to detect deep central mediastinal lymph nodes long before a displacement of pleuromediastinal lines occurs on radiograph. Majority of mediastinal Hodgkin and non-Hodgkin lymphomas are homogeneously hypoechoic. Enlarged lymphatic nodes are hypoechoic and fuse to form large masses leading to displacement or engulfing mediastinal structures. Sometimes parasternal infiltration of the thoracic wall, compression of the superior vena cava, pleural effusion including chylothorax can be seen due to presence of large tumours.

Percutaneous biopsy under US guidance has very good diagnostic accuracy and many other advantages, without irradiation implied by CT approach. US-guided biopsy with cutting needles offers better samples compared with fine needle aspiration allowing histological diagnosis with subtyping. ${ }^{10}$ However, mediastinal lymphomas may be isoechoic on hyperechoic, relative to surrounding tissue, at primary manifestation. In bulky disease, several adjacent nodes fuse to form a single, larger mass of heterogeneous echogenicity. ${ }^{11}$ Old parasternal sonography is a sensitive technique for the detection of tumours in the anterior mediastinal and subcarinal mediastinal spaces. ${ }^{12}$ Ultrasound is used to evaluate any abnormality in patients presenting with a widened superior mediastinum on chest radiography. Ultrasound can also be used for evaluating superior mediastinal masses, for the evaluation of lymphadenopathy in cervical, supraclavicular and axillary areas, for follow up of known cases such as lymphoma, for monitoring response to treatment. ${ }^{13}$ Lymph nodes in NHL are larger as compared with those in HL and have a predilection for non-contiguous and / or haematogenous spread to thoracic and distant nodal and extranodal sites. ${ }^{5}$ Large cell mediastinal lymphoma is locally invasive and bulky malignancy which occurs more commonly in young women. Full dose CHOP (Cyclophosphamide, Doxorubicin, Vincristine, Prednisone) chemotherapy and radiation is used for curing the disease of low or moderate bulk, but bulky disease requires intense dedicated treatment. 13

Resection is done in benign-appearing lesions. Malignantappearing lesions are biopsied by the least invasive route. ${ }^{14}$ On CT, NHL is seen as a discrete anterior mediastinal mass with a contour that is lobulated. The tumour demonstrates homogeneous soft-tissue attenuation most commonly, although large lymph nodal masses may show heterogeneity with complex low attenuation indicating necrosis, haemorrhage, or cystic degeneration. ${ }^{4}$ Large B-cell lymphoma and lymphoblastic lymphoma are the most common subtypes of NHL that primarily involves the anterior mediastinum. 
Primary mediastinal large B-cell lymphomas are seen as large and lobulated anterior mediastinal masses and occur most commonly in young adults with an average age of 26 years Also they often show direct invasion of adjacent structures. Lymphoblastic lymphomas are highly aggressive. High-grade lymphomas, arising from thymic lymphocytes in $16 \%$ of patients with NHL may have pleural involvement. They may present as a solitary nodule or multiple, broad-based pleural mass, or a combination of the two, and pleural effusion. Pleural involvement (which is frequently overlooked on conventional imaging) should be adequately identified because that finding dictates patient management. Unrecognized and untreated pleural involvement is associated with increased risk of treatment failure. ${ }^{4}$

\section{CONCLUSIONS}

Ultrasound can provide useful information in the complex assessment of the mediastinal masses occurring in the anterior (prevascular) and posterior compartments of the mediastinum. It offers also the possibility of guiding biopsies in those clinical scenarios, with many advantages over CT guidance. High-resolution suprasternal sonography is lowcost modality that is easy to perform and is an effective diagnostic tool in evaluating the findings in the paratracheal region and aortopulmonary window.

Financial or other competing interests: None.

Disclosure forms provided by the authors are available with the full text of this article at jemds.com.

\section{REFERENCES}

[1] Shields TW, LoCicero J, Reed CE, et al. General thoracic surgery. Vol 1. Lippincott Williams \& Wilkins Publication 2009.
[2] Duwe BV, Sterman DH, Musani AI. Tumors of the mediastinum. Chest 2005;128(4):2893-909.

[3] Frampas E. Lymphomas: basic points that radiologists should know. Diagn Interv Imaging 2013;94(2):131-44.

[4] Bae YA, Lee KS. Cross-sectional evaluation of thoracic lymphoma. Radiol Clin North Am 2008;46(2):253-64.

[5] Lichtenstein AK, Levine A, Taylor CR, et al. Primary mediastinal lymphoma in adults. Am J Med 1980;68(4):509-14.

[6] Wernecke K, Vassallo P, Hoffmann G, et al. Value of sonography in monitoring the therapeutic response of mediastinal lymphoma: comparison with chest radiography and CT. AJR Am J Roentgenol 1991;156(2):265-72.

[7] Wernecke K, Peters PE, Galanski M. Mediastinal tumors: evaluation with suprasternal sonography. Radiology 1986;159(2):405-9.

[8] Silverman NA, Sabiston DC. Mediastinal masses. Surg Clin North Am 1980;60(4):757-77.

[9] Wernecke K, Diederich S. Sonographic features of mediastinal tumors. AJR Am J Roentgenol 1994;163(6):1357-64.

[10] Chira RI, Chira A, Mircea PA, et al. Mediastinal massestransthoracic ultrasonography aspects. Medicine (Baltimore) 2017;96(49):e9082.

[11] Wernecke K, Potter R, Peters PE, et al. Parasternal mediastinal sonography: sensitivity in the detection of anterior mediastinal and subcarinal tumors. AJR Am J Roentgenol 1988;150(5):1021-6.

[12] Ganesan S. Sonographic approach to the superior mediastinum. Indian J Radiol Imaging 2001;11(2):71-3.

[13] Jacobson JO, Aisenberg AC, Lamarre L, et al. Mediastinal large cell lymphoma. An uncommon subset of adult lymphoma curable with combined modality therapy. Cancer 1988;62(9):1893-8.

[14] Wright CD, Mathisen DJ. Mediastinal tumors: diagnosis and treatment. World J Surg 2001;25(2):204-9. 